\title{
Combination of HAI-FUDR and Systemic Gemcitabine and Cisplatin in Unresectable Cholangiocarcinoma: A Dose Finding Single Center Study
}

\author{
Heike Pietge ${ }^{a}$ Patricia Sánchez-Velázquez ${ }^{b}$ Dilara Akhoundova ${ }^{a} \quad$ Alexander Siebenhüner $^{a}$ \\ Thomas Winder $^{\text {a Helga Bachmann }}{ }^{\text {a }}$ Thi Dan Linh Nguyen-Kim ${ }^{c}$ Stefan Breitenstein ${ }^{d}$ \\ Alexander Knuth $^{a}$ Henrik Petrowsky ${ }^{b}$ Bernhard Pestalozzi ${ }^{a}$ Pierre-Alain Clavien ${ }^{b}$ \\ Panagiotis Samaras ${ }^{\text {a }}$ \\ aDepartment of Medical Oncology and Hematology, Division of Medical Oncology, University Hospital Zurich, \\ Zurich, Switzerland; b Department of General Surgery and Transplantation, University Hospital Zurich, Zurich, \\ Switzerland; 'Department of Radiology, University Hospital Zurich, Zurich, Switzerland; 'Department of Surgery, \\ Kantonsspital Winterthur, Winterthur, Switzerland
}

\section{Keywords}

Cholangiocarcinoma - Unresectable cholangiocarcinoma . Intrahepatic cholangiocarcinoma $\cdot$ Hepatic arterial infusion . Floxuridine · Intrahepatic chemotherapy

\begin{abstract}
Background: Unresectable cholangiocarcinoma has a poor prognosis and treatment options are limited. Combined systemic and intrahepatic chemotherapy may improve local control and enable downsizing. The aim of this study was to determine the maximum tolerated dose (MTD) of intravenous gemcitabine combined with intravenous cisplatin and hepatic arterial infusion (HAI) with floxuridine (FUDR) in patients with unresectable intrahepatic or hilar cholangiocarcinoma. Methods: Twelve patients were treated within a $3+$ 3 dose escalation algorithm with 600,800 , or $1,000 \mathrm{mg} / \mathrm{m}^{2}$ gemcitabine and predefined doses of cisplatin $25 \mathrm{mg} / \mathrm{m}^{2}$ on days 1 and 8 , q21, for 4 cycles, and FUDR $0.2 \mathrm{mg} / \mathrm{kg}$ on days 1-14 as continuous HAl, q28, for 3 cycles. Safety and toxicity as well as resectability rates after 3 months and preliminary survival data are reported. Results: The determined MTD for
\end{abstract}

karger@karger.com www.karger.com/ocl

Karger $\stackrel{\text { ' }}{5}$

GOPEN ACCESS
(C) 2021 The Author(s)

Published by S. Karger AG, Basel

This is an Open Access article licensed under the Creative Commons Attribution-NonCommercial-4.0 International License (CC BY-NC) (http://www.karger.com/Services/OpenAccessLicense), applicable to the online version of the article only. Usage and distribution for commercial purposes requires written permission. gemcitabine was $800 \mathrm{mg} / \mathrm{m}^{2}$. Dose limiting toxicities were neutropenic fever and biliary tract infections. In total, $27 \%$ of the patients showed partial remission and $73 \%$ stable disease. Although none of the patients achieved resectability after 3 months, the 3-year overall survival rate was 33\%, median overall survival 23.9 months (range 1-49), and median progression-free survival 10.1 months (range 2-40). Conclusions: Intravenous gemcitabine/cisplatin plus HAI-FUDR is feasible and appears effective for disease control. Larger prospective studies evaluating this triplet combination are warranted.

(c) 2021 The Author(s) Published by S. Karger AG, Basel

\section{Introduction}

Cholangiocarcinoma (CCA) arises from the biliary epithelium of intra- and extrahepatic bile ducts. The incidence of intrahepatic CCA has increased markedly in the

ClinicalTrials.gov Identifier: NCT01692704, registered on September 25, 2012. 
last decades, while the incidence of extrahepatic CCA has increased only modestly [1]. CCA is associated with a poor prognosis, and mortality rates also seem to have increased during the last decades, according to registry data [2]. Surgery offers the only chance for long-term survival for CCA patients, but it is curative only in the minority of all cases, with a 9-18\% 5-year survival rate for hilar/intrahepatic CCA and 20-30\% for distal lesions [3]. Only a few selected patients with localized hilar CCA qualify for a multimodality treatment, eventually followed by a liver transplantation. This approach may lead to 5-year survival rates of up to $80 \%$, according to the Mayo Clinic experience [4].

However, the disease is often locally too advanced to allow for complete resection. Therefore, strategies for downsizing intrahepatic or hilar CCA confined to the liver without extrahepatic spread are needed. The ABC-02 phase III trial established the current standard of systemic chemotherapy in advanced biliary tract cancer, but it showed an only modest survival benefit for patients treated with gemcitabine-cisplatin compared to those treated with gemcitabine monotherapy (11.7 vs. 8.1 months) [5]. Konstantinidis et al. [6] presented promising data with hepatic arterial infusion (HAI) chemotherapy by using floxuridine (FUDR) in patients with unresectable CCA in a retrospective analysis. Objective response rates of up to $59 \%$ and a prolonged overall survival (OS) with combined HAI and systemic chemotherapy compared to systemic treatment alone have been observed retrospectively $(30.8$ vs. 18.4 months; $p<0.001$ ) [6, 7]. Recently, the combination of HAI with FUDR plus systemic gemcitabine and oxaliplatin was shown to be well tolerated and active in a phase II trial [8].

Selective HAI to the liver has been available for more than four decades, and is currently under evaluation for different tumor entities and clinical settings [6, 9-14]. Whilst blood supply to the liver parenchyma arises from both the hepatic artery and the portal vein, most hepatic tumors, including CCA, are almost exclusively perfused by the hepatic artery. FUDR is an active metabolite of 5-fluorouracil and has a $90 \%$ extraction rate within the liver on the first pass. Local infusion of FUDR into the hepatic artery will therefore expose the tumor to high drug concentrations with minimal systemic exposure [5]. The safety of FUDR and the benefit of HAI have been demonstrated predominantly in patients with colorectal liver metastases, either as a downsizing strategy or as adjuvant treatment after resection of colorectal metastases [14-16].

Preclinical data indicate synergistic activity of FUDR and gemcitabine in colon cancer cells. Application of both drugs led to more than additive growth inhibition and interference with colony formation in HT-29 colon cancer cells [17]. No preclinical data exist on the application of all three drugs combined. In light of these sparse data, we decided to combine these two treatment modalities - HAI with FUDR and systemic chemotherapy with cisplatin and gemcitabine - to develop a new downsizing strategy exclusively for patients with locally advanced, unresectable intrahepatic or hilar CCA without distant metastases who are not candidates for liver transplantation. Gemcitabine was the preferred systemic combination partner with FUDR in the retrospective study of Konstantinidis et al. [6] mentioned above, but data on drug dosing and modifications were not reported. Cisplatin and FUDR have been studied in the past as combined HAI, and this double combination seems to be feasible. Taken together, both gemcitabine and cisplatin have been combined each with FUDR without excessive toxicities reported, but safety data on the administration of all three drugs together are lacking. Since FUDR has been shown to be safe and efficacious, we kept the established HAI dose and chose gemcitabine for dose escalation because of the rather high standard dose of $1,000 \mathrm{mg} / \mathrm{m}^{2}$ usually administered intravenously compared to cisplatin. This clinical study is therefore a "first-in-human" treatment approach.

\section{Subjects and Methods}

\section{Patients}

In this open-label, prospective, nonrandomized, single-arm doseescalation study(ClinicalTrials.govIdentifier:NCT01692704), 12 patients with unresectable CCA were included between June 2012 and January 2016 at the University Hospital Zurich, Switzerland. The trial design is depicted in Figure 1.

The main inclusion criteria were: (1) having nonresectable, histologically or cytologically proven cholangiocellular adenocarcinoma including gallbladder cancer; (2) being no candidate for liver transplantation; (3) having no extrahepatic tumor spread, as evaluated by PET-CT (positron emission tomography combined with computed tomography) of the chest and the abdomen/pelvis with the exception of limited indetermined and potentially resectable small lung nodules or hilar lymph node involvement; and (4) having adequate liver function and kidney function test results, including bilirubin $<2 \times$ the upper limit of normal (ULN), aspartate aminotransferase $<5 \times$ ULN, alanine aminotransferase $<5 \times$ ULN, and alkaline phosphatase $<5 \times$ ULN. The main exclusion criteria were: (1) an anatomic variant in the arteriogram, which prevents selective delivery of chemotherapy to the liver, and (2) a life expectancy $<3$ months.

The purpose of this study was to evaluate the safety and toxicity of this combined regional and systemic treatment over a treatment period of 3 months. Patients were allowed to continue this triplet treatment after the end of the 3-month study period if it was tolerated and efficacious. The primary objective was the definition 


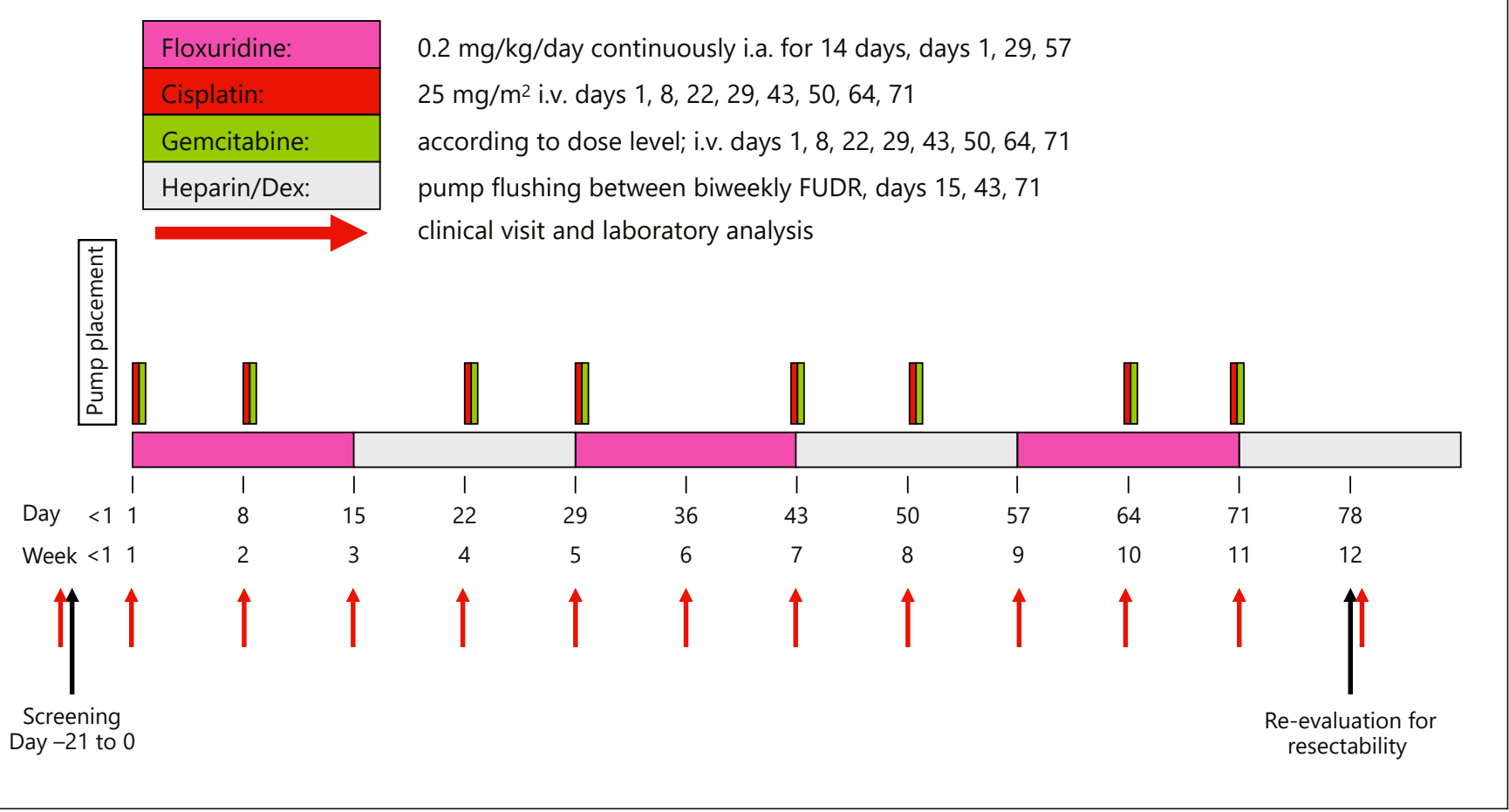

Fig. 1. Trial overview. FUDR, floxuridine; Dex, dexamethasone.

of the maximum tolerated dose (MTD) of intravenous gemcitabine in combination with intravenous cisplatin and intra-arterial FUDR. Secondary objectives were: (1) the response rate, using Response Evaluation Criteria in Solid Tumors (RECIST 1.1) after 3 months of chemotherapy; (2) the rate of conversion to resectability; (3) surgical complications; (4) the toxicity of the chemotherapy according to the Common Terminology Criteria for Adverse Events (CTCAE) v4.03 reporting system; (5) progression-free survival (PFS); and (6) OS. Surgical complications were ranked according to the Clavien-Dindo classification with calculation of the Comprehensive Complication Index (CCI) $[18,19]$. This system ranks complications by severity according to their therapeutic consequences. Thus, grade I and II complications are events requiring only bedside procedures or pharmacologic treatments, whereas grade III complications require surgical, radiological, or endoscopic treatment. Grade IV complications are life-threatening complications, and grade $\mathrm{V}$ complications correspond to death. The CCI is based on the complication grading of the Clavien-Dindo classification and implements every occurred complication after an intervention. The overall morbidity is reflected on a scale from 0 ("no complication") to 100 ("death").

\section{Administration Technique for HAI-FUDR}

For the intra-arterial administration of FUDR, we used a totally implantable pump device (CODMAN 3000 implantable infusion pump; Codman \& Shurtleff Inc.) with a refillable reservoir. During a laparotomy, the hepatoduodenal ligament was approached and the gastroduodenal artery (GDA) dissected and encircled. The pump catheter was inserted into the GDA at its junc- tion with the common hepatic artery. A separate, transverse skin incision was made in the right lower abdominal quadrant, and a subcutaneous pocket was tailored. The pump was placed into this pocket and fixed to the fascia to avoid dislocation of the device. Appropriate placement of the catheter in the GDA was confirmed during laparotomy by infusion of fluorescein into the pump and evaluating the liver parenchyma with a hand-held Woods lamp. Finally, the gallbladder was resected and the abdomen was closed.

\section{Regional HAI-FUDR Chemotherapy}

The patients received FUDR $0.2 \mathrm{mg} / \mathrm{kg} /$ day for 14 days in combination with a total of $20 \mathrm{mg}$ dexamethasone and a total of 50,000 units of heparin diluted in $\mathrm{NaCl} 0.9 \%$ to a total volume of $30 \mathrm{~mL}$ into the pump reservoir, repeated at day 29 , for a total of 3 cycles. The pump reservoir was filled with $30 \mathrm{~mL}$ saline and 50,000 units of heparin for days 15-28 of each cycle to maintain a continuous efflux and thus avoid catheter occlusion.

\section{Systemic Chemotherapy}

Cisplatin $25 \mathrm{mg} / \mathrm{m}^{2}$ was administered as intravenous infusion at days 1 and 8, repeated on day 22, for a total of 4 cycles. Gemcitabine was given according to the dose level the patient was treated with, i.e., $600 \mathrm{mg} / \mathrm{m}^{2}$ (dose level 1), $800 \mathrm{mg} / \mathrm{m}^{2}$ (dose level 2), or $1,000 \mathrm{mg} / \mathrm{m}^{2}$ (dose level 3) as intravenous infusion at days 1 and 8 , repeated on day 22 , for a total of 4 cycles.

\section{Dose Escalation}

A traditional dose level escalation set-up was used to define the MTD for gemcitabine following a $3+3$ algorithm. The first cohort 
Table 1. Patients' characteristics at baseline $(n=12)$

\begin{tabular}{|c|c|}
\hline Median age (range), years & $63.5(33-72)$ \\
\hline \multicolumn{2}{|l|}{ Gender, $n(\%)$} \\
\hline Male & $9(75)$ \\
\hline Female & $3(25)$ \\
\hline \multicolumn{2}{|l|}{ Primary tumor, $n(\%)$} \\
\hline Intrahepatic & $9(75)$ \\
\hline Hilar & $2(17)$ \\
\hline Gallbladder & $1(8)$ \\
\hline \multicolumn{2}{|l|}{ Nodal involvement, $n(\%)$} \\
\hline cN0 & $8(67)$ \\
\hline $\mathrm{cN}+$ & $4(33)$ \\
\hline \multicolumn{2}{|l|}{ Grade, $n(\%)$} \\
\hline G1 & $0(0)$ \\
\hline G2 & $1(8)$ \\
\hline G3 & $5(42)$ \\
\hline Unknown & $6(50)$ \\
\hline \multicolumn{2}{|l|}{$\mathrm{ECOG}, n(\%)$} \\
\hline 0 & $3(25)$ \\
\hline 1 & $9(75)$ \\
\hline Previous chemotherapy, $n(\%)$ & $2(17)$ \\
\hline \multicolumn{2}{|l|}{ Liver function tests } \\
\hline ALT abnormal, $n$ (range in U/L) (\%) & $7(64-168)(58)$ \\
\hline AST abnormal, $n$ (range in U/L) (\%) & $9(71-174)(75)$ \\
\hline Bilirubin abnormal, $n$ & \\
\hline (range in umol/L) (\%) & $2(3-41)(17)$ \\
\hline
\end{tabular}

of 3 patients received gemcitabine at dose level $1\left(600 \mathrm{mg} / \mathrm{m}^{2}\right)$ in combination with cisplatin and FUDR. Once all 3 patients at a given dose level had received at least 2 applications of systemic chemotherapy and 1 out of these 3 patients had received 4 applications of systemic chemotherapy and if no dose limiting toxicity (DLT) occurred, the next 3 patients were included at the nexthigher dose level. If 1 out of a cohort of 3 patients experienced a DLT, 3 additional patients were recruited at the same dose level. If no further DLT were experienced in this cohort of 6 patients, the dose was escalated to the next higher level for subsequent patients. If a second DLT was observed at the same dose level, all further patients would have been deescalated to the previous dose level.

\section{Maximum Tolerated Dose}

The MTD of gemcitabine in combination with fixed doses of cisplatin and FUDR was defined as one dose level below the dose level that caused DLTs in more or equal than one-third of patients ( 2 or more in a cohort of 6 patients), as described above $(3+3$ algorithm).

\section{Dose Limiting Toxicity}

A DLT was defined as an adverse event (AE) or laboratory abnormality that fulfilled one of the following criteria during the first 4 applications of systemic treatment: (1) grade 4 neutropenia lasting $\geq 7$ days or febrile neutropenia; (2) grade 4 thrombocytopenia; (3) grade 3 or 4 gastrointestinal symptoms (diarrhea, vomiting, or mucositis) which were not reduced to grade 1 within 7 days of appropriate supportive care; (4) grade 3/4 skin toxicity, for example, rash or hand-foot syndrome; (5) grade $3 / 4$ pulmonary toxicity, for

Systemic and Intra-Arterial

Chemotherapy for Cholangiocarcinoma example, dyspnea, edema, or alveolitis, which did not resolve to grade 1 within 3 days after gemcitabine cessation; (6) grade 3/4 elevation of liver transaminases despite discontinuation of HAIFUDR; (7) any grade 3/4 toxicity which was considered to be dose limiting after discussion between the investigators and the sponsor.

\section{Statistical Methods}

All statistical analyses were performed using the SPSS software package, version 20.0 (Chicago, IL, USA). PFS and OS analyses were performed using the Kaplan-Meier method. PFS was defined as the time from start of chemotherapy to radiologically confirmed progression or death from any cause, whichever occurred first. OS was defined as the time from start of chemotherapy until death from any cause. Patients alive at the time of evaluation were censored at the last follow-up (the cut-off date was April 30, 2018).

\section{Results}

\section{Patient Characteristics}

In total, 12 patients $\geq 18$ years were enrolled in the study. The majority of the patients had intrahepatic CCA (75\%), and hilar CCA or gallbladder cancer was present in 17 and $8 \%$ of the patients, respectively. Poor risk factors, such as poorly differentiated tumors and lymph node involvement, were documented in 42 and 33\%, respectively. The majority of the patients $(83 \%)$ were treatment naïve. Detailed patient characteristics at baseline are listed in Table 1.

\section{Dose Escalation and Toxicity Including DLT and MDT}

The initial dose for cohort $1(n=3)$ was gemcitabine $600 \mathrm{mg} / \mathrm{m}^{2}$ on days 1 and 8 intravenously every 3 weeks. Cisplatin $25 \mathrm{mg} / \mathrm{m}^{2}$ on days 1 and 8 intravenously every 3 weeks and FUDR $0.2 \mathrm{mg} / \mathrm{kg}$ body weight on days $1-14$ as continuous hepatic intra-arterial infusion every 4 weeks were administered per protocol. No DLTs and no grade 4 toxicities occurred at this dose level.

Dose escalation of gemcitabine to $800 \mathrm{mg} / \mathrm{m}^{2}$ on days 1 and 8 intravenously every 3 weeks was performed as scheduled in dose level $2(n=3)$. Cisplatin and FUDR were administered at their predefined doses as before. At this dose level as well, no DLTs and no grade 4 toxicities occurred.

At dose level $3(n=3)$, gemcitabine was escalated to $1,000 \mathrm{mg} / \mathrm{m}^{2}$ on days 1 and 8 intravenously every 3 weeks, while the doses of cisplatin and FUDR remained unchanged. At this level, we observed grade 4 toxicities in 2 out of 3 patients (cholangiosepsis, non-gastrointestinalrelated sepsis, and lymphopenia), with 1 DLT due to recurrent cholangiosepsis despite discontinuation of 
Table 2. Treatment-related adverse events across all patients and dose level cohorts

\begin{tabular}{lllll}
\hline & Overall, & $\mathrm{G} 1 / 2$, & $\mathrm{G} 3$, & $\mathrm{G} 4$, \\
& $n(\%)$ & $n(\%)$ & $n(\%)$ & $n(\%)$ \\
\hline Hematological toxicity & & & & \\
Anemia & $9(75)$ & $5(42)$ & $4(33)$ & 0 \\
Thrombopenia & $9(75)$ & $7(58)$ & $2(17)$ & 0 \\
Lymphopenia & $7(58)$ & $4(33)$ & $2(17)$ & $1(8)$ \\
Leukopenia & $7(58)$ & $3(25)$ & $3(25)$ & $1(8)$ \\
Neutropenia & $6(50)$ & $2(17)$ & $4(33)$ & 0 \\
Gastrointestinal and hepatic toxicity & & & & 0 \\
Nausea & $6(50)$ & 50 & 0 & 0 \\
Anorexia & $5(42)$ & $5(42)$ & 0 & 0 \\
AST increase & $4(33)$ & $2(17)$ & $2(17)$ & 0 \\
AP increase & $3(25)$ & $1(8)$ & $2(17)$ & 0 \\
ALT increase & $2(17)$ & $1(8)$ & $1(8)$ & $1(8)$ \\
GGT increase & $2(17)$ & 0 & $1(8)$ & $1(8)^{*}$ \\
BLT infection & $2(17)$ & $1(8)$ & 0 & 0 \\
Constipation & $2(17)$ & $2(17)$ & 0 & 0 \\
Diarrhea & $2(17)$ & $2(17)$ & 0 & 0 \\
Gastritis & $2(17)$ & $2(17)$ & 0 & 0 \\
Duodenal ulceration & $1(8)$ & $1(8)$ & 0 & 0 \\
Others & & & & 0 \\
Fatigue & $8(67)$ & $4(33)$ & $4(33)$ & 0 \\
Infections other than BLT & $4(33)$ & $1(8)$ & $2(17)$ & $1(8)^{*}$ \\
Hyponatremia & $3(25)$ & $3(25)$ & 0 & 0 \\
Tinnitus & $2(17)$ & $2(17)$ & 0 & 0 \\
Paresthesia & $2(17)$ & $2(17)$ & 0 & 0 \\
Fever & $2(17)$ & $2(17)$ & 0 & 0 \\
\hline
\end{tabular}

AP, alkaline phosphatase; ALT, alanine aminotransferase; AST, aspartate aminotransferase; BLT, biliary tract; GGT, $\gamma$-glutamyltransferase. * Dose limiting toxicity.

FUDR. One patient died 6 weeks after study enrollment due to a pulmonary embolism. This patient already had had an underlying thromboembolic disease before study enrollment due to a tumor-related thrombophilia. Therefore, this grade 5 complication was not considered to be study drug related and not classified as a DLT.

Because of the aforementioned 1 DLT in cohort 3, we continued with an expansion cohort $(n=3)$ at the same dose of gemcitabine $1,000 \mathrm{mg} / \mathrm{m}^{2}$ on days 1 and 8 intravenously every 3 weeks and cisplatin and FUDR at their predefined doses. In the expansion cohort, we observed a grade 4 toxicity in 1 patient (neutropenic fever), which was classified as a second DLT per protocol.

In total, 2 DLTs occurred at dose level 3 with gemcitabine $1,000 \mathrm{mg} / \mathrm{m}^{2}$. As a consequence, the MTD of gemcitabine was defined as $800 \mathrm{mg} / \mathrm{m}^{2}$ on days 1 and 8 intravenously every 3 weeks, in combination with cisplatin $25 \mathrm{mg} / \mathrm{m}^{2}$ on days 1 and 8 intravenously every 3 weeks and FUDR $0.2 \mathrm{mg} / \mathrm{kg}$ body weight on days $1-14$ as continuous intra-arterial hepatic infusion every 4 weeks.

\section{AEs due to Study Drug Treatment}

Grade 3/4 AEs were dose dependent and occurred mainly at dose level 3 with gemcitabine $1,000 \mathrm{mg} / \mathrm{m}^{2}$ (Tables 2,3$)$. The most frequent grade $3 / 4$ AEs were anemia and neutro-, lympho- and thrombocytopenia, as well as liver function test elevation and fatigue.

In total, $67 \%$ of the patients completed the preplanned 3 months of study treatment, and $33 \%(n=4)$ of the patients did not: 1 patient developed recurrent biliary tract infections defined as DLTs and had to stop treatment, 1 patient died 6 weeks after study enrollment following pulmonary embolism due to an underlying thromboembolic disease, and 2 patients stopped for other reasons (1 due to psychiatric disorder and 1 due to non-study-drug-related biliary tract infection).

Two out of all 12 patients (17\%) experienced a dose delay and dose reductions due to toxicity of systemic and intrahepatic chemotherapy. One patient in cohort 3 (gemcitabine $1,000 \mathrm{mg} / \mathrm{m}^{2}$ ) had recurrent biliary tract infections requiring treatment discontinuation; the other 
Table 3. Grade 3/4 AEs

a Patients per cohort with treatment-related grade $3 / 4$ toxicities

\begin{tabular}{|c|c|c|c|c|c|c|c|c|c|c|}
\hline \multirow[t]{2}{*}{ Cohort } & \multirow[t]{2}{*}{$\begin{array}{l}\text { Gemcitabine } \\
\text { dose, } \mathrm{mg} / \mathrm{m}^{2}\end{array}$} & \multirow[t]{2}{*}{$\begin{array}{l}\text { Patients, } \\
n\end{array}$} & \multicolumn{2}{|c|}{ Hematological toxicity, $n$} & \multicolumn{2}{|c|}{$\begin{array}{l}\text { Gastrointestinal and } \\
\text { hepatic toxicity, } n\end{array}$} & \multicolumn{2}{|c|}{ Other toxicity, $n$} & \multirow[t]{2}{*}{$\begin{array}{l}\text { DLTs, } \\
n\end{array}$} & \multirow[t]{2}{*}{$\begin{array}{l}\text { SAEs, } \\
n\end{array}$} \\
\hline & & & G3 & G4 & G3 & G4 & G3 & G4 & & \\
\hline 1 & 600 & 3 & 1 & 0 & 1 & 0 & 0 & 0 & 0 & 0 \\
\hline 2 & 800 & 3 & 1 & 0 & 1 & 0 & 0 & 0 & 0 & 4 \\
\hline 3 & 1,000 & 3 & 1 & 1 & 2 & 1 & 3 & 2 & 1 & 10 \\
\hline 3 exp. & 1,000 & 3 & 3 & 1 & 2 & 0 & 2 & 0 & 1 & 2 \\
\hline Total & & 12 & & & & & & & 2 & 16 \\
\hline
\end{tabular}

b All treatment-related grade 3/4 toxicities occurring per cohort

\begin{tabular}{|c|c|c|c|c|c|c|c|c|c|c|}
\hline \multirow[t]{2}{*}{ Cohort } & \multirow[t]{2}{*}{$\begin{array}{l}\text { Gemcitabine } \\
\text { dose, } \mathrm{mg} / \mathrm{m}^{2}\end{array}$} & \multirow[t]{2}{*}{$\begin{array}{l}\text { Patients, } \\
n\end{array}$} & \multicolumn{2}{|c|}{ Hematological AEs, $n$} & \multicolumn{2}{|c|}{$\begin{array}{l}\text { Gastrointestinal and } \\
\text { hepatic AEs, } n\end{array}$} & \multicolumn{2}{|c|}{ Other toxicity AEs, $n$} & \multirow[t]{2}{*}{$\begin{array}{l}\text { DLTs, } \\
n\end{array}$} & \multirow[t]{2}{*}{$\begin{array}{l}\text { SAEs, } \\
n\end{array}$} \\
\hline & & & G3 & G4 & G3 & G4 & G3 & G4 & & \\
\hline 2 & 800 & 3 & 1 & 0 & 1 & 0 & 0 & 0 & 0 & 4 \\
\hline 3 & 1,000 & 3 & 3 & 1 & 4 & 2 & 3 & 2 & 1 & 10 \\
\hline 3 exp. & 1,000 & 3 & 8 & 1 & 2 & 0 & 2 & 0 & 1 & 2 \\
\hline
\end{tabular}

AE, adverse event; DLT, dose limiting toxicity; SAE, serious adverse event.

patient (expansion cohort, gemcitabine $1,000 \mathrm{mg} / \mathrm{m}^{2}$ ) had neutropenic fever due to an enterocolitis, which required a $25 \%$ dose reduction. Both were determined as DLTs.

In total, 16 serious AEs (SAEs) occurred during the study period; 10 SAEs were possibly or likely related to study drug treatment, and in all cases this was due to infections requiring hospitalization (pneumonia, biliary tract infection, and enterocolitis). Other SAEs that were not related to study drug treatment were postoperative pain after pump insertion, biliary tract infection that had been present before initiation of study drug treatment, an episode of depression that was related to a psychiatric disorder that had been present before study enrollment, and a pulmonary embolism in a patient with underlying thromboembolic disease. One patient developed a volvulus of the colon as described below.

\section{No SAEs Were Considered as Suspected Unexpected \\ Serious Adverse Reactions}

All reported SAEs were within the expected range and were consistent with the known adverse reactions to the given therapy. There has been no change in the perceived risk of the trial treatment identified from the cumulative safety data. No new safety issues were identified.
Table 4. Surgical complications after pump implantation

\begin{tabular}{|c|c|c|c|}
\hline Patient & Complication(s) & $\begin{array}{l}\text { Clavien-Dindo } \\
\text { classification }\end{array}$ & CCI \\
\hline 1 & Anaphylaxis & II & 20.9 \\
\hline 2 & Seroma & I & 8.7 \\
\hline 3 & $\begin{array}{l}\text { Seroma } \\
\text { Dislocation of the pump }\end{array}$ & $\begin{array}{l}\text { I } \\
\text { IIIb }\end{array}$ & 8.7 \\
\hline 4 & $\begin{array}{l}\text { Edema limbs } \\
\text { Wound dehiscence } \\
\text { Volvulus, pump related }\end{array}$ & $\begin{array}{l}\text { I } \\
\text { I } \\
\text { IIIb }\end{array}$ & 35.9 \\
\hline 5 & Seroma & I & 8.7 \\
\hline 6 & - & - & 0 \\
\hline 7 & Cholangitis & II & 20.9 \\
\hline 8 & Seroma & I & 8.7 \\
\hline 9 & - & - & 0 \\
\hline 10 & Seroma & I & 8.7 \\
\hline 11 & Seroma & I & 8.7 \\
\hline 12 & $\begin{array}{l}\text { Seroma } \\
\text { Hematoma }\end{array}$ & $\begin{array}{l}\text { I } \\
\text { I }\end{array}$ & 12.2 \\
\hline
\end{tabular}

CCI, Comprehensive Complication Index. 
Fig. 2. Response assessment according to RECIST 1.1 after 3 months of treatment. *, patients with hilar cholangiocarcinoma; +, patient with gallbladder and liver parenchyma infiltration; GEM, gemcitabine.

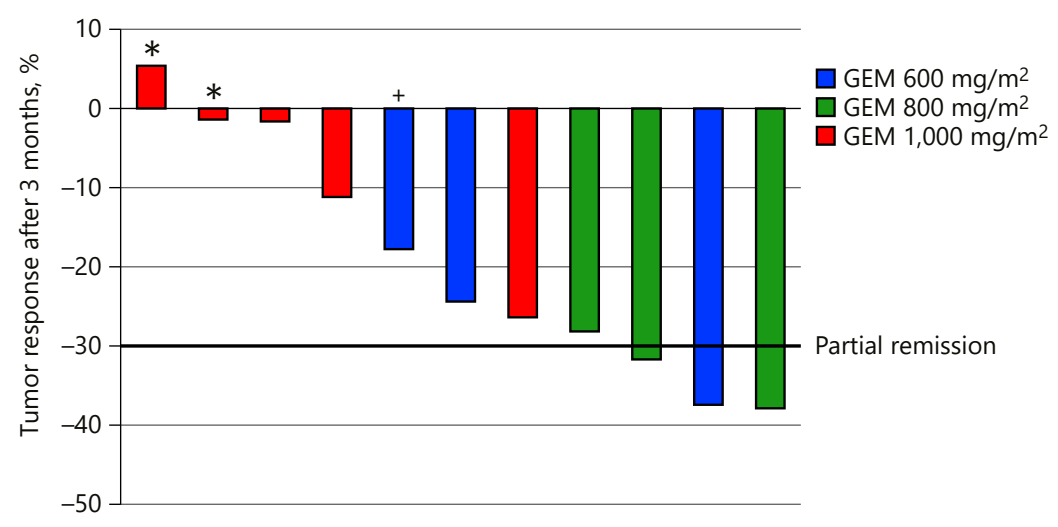

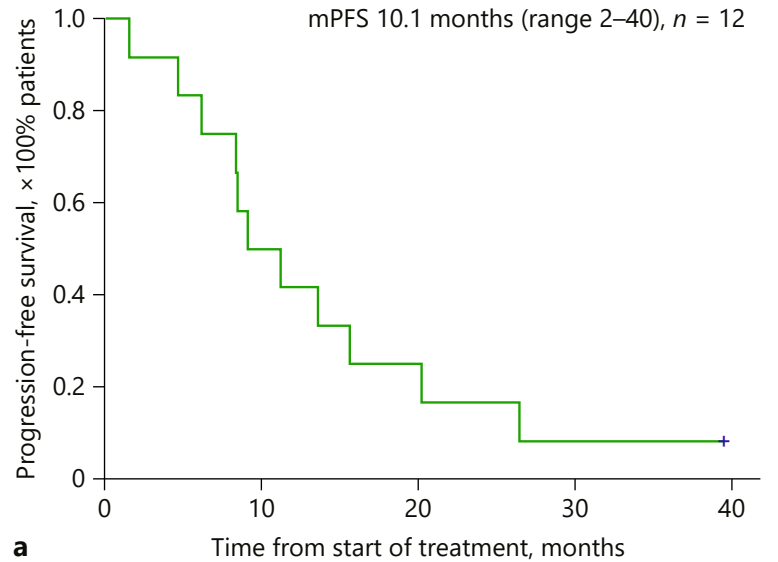

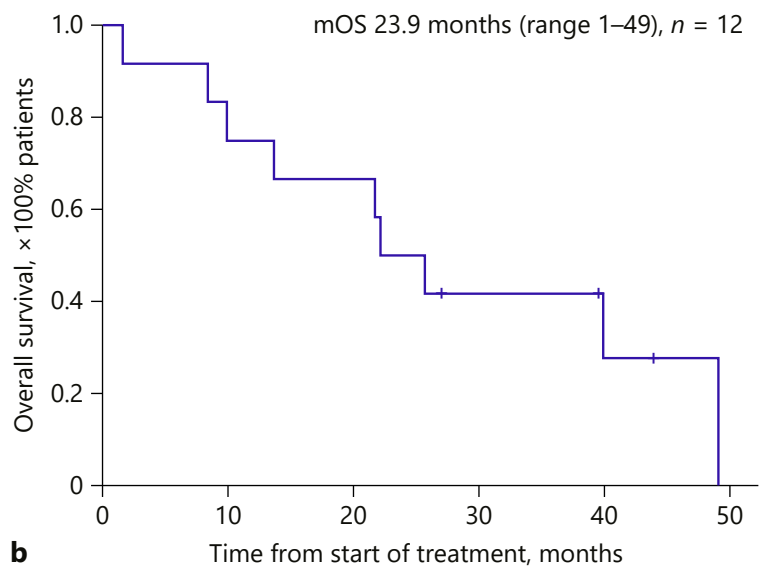

Fig. 3. Median progression-free survival (mPFS; a) and median overall survival (mOS; b) curves with unresectable cholangiocarcinoma. PFS was defined as the time from start of chemotherapy until disease progression or death from any cause. OS was defined as the time from start of chemotherapy until death from any cause. Vertical lines indicate that patients' data were censored.

\section{Pump-and Catheter-Related Complications}

A total of $83.3 \%$ of the patients presented with mild complications after initial surgery (Clavien-Dindo IIIIa) up to discharge, with a median CCI of 12.2 (Table 4). Major pump- or catheter-related complications occurred only in 2 patients (16.7\%). In both cases these were classified as Clavien-Dindo IIIb complications, as they required a surgical reintervention. One patient developed a volvulus of the colon 8 months after pump implantation, due to strangulation of the pump catheter, requiring surgery and stop of the treatment. The other patient had a dysfunction of the pump catheter system due to a broken metal insertion at the pump container that occurred 3 months after treatment. The pump had to be removed. The median time from pump placement to the start of chemotherapy was 10 days (range 4-27).

Response Assessment and Resection Rate after 3 Months

Response assessment according to RECIST 1.1 was performed after 3 months of treatment with CT/PET-CT scans. In total, a complete response, partial response, and stable disease was achieved in $0,3(27 \%)$, and $8(73 \%)$ patients, respectively (Fig. 2). None of the patients had progressive disease at that time. One patient was excluded from the analysis (not applicable) due to early death after 6 weeks due to a pulmonary embolism. Only patients with intrahepatic CCA showed a partial response according to RECIST 1.1. Both patients with hilar CCA had no objective response of the tumor. The time to best response was 4.6 months (range 2-26).

None of the patients was resectable after 3 months of study drug treatment according to multidisciplinary tumor board decision. 


\section{PFS and OS}

Median follow-up from start of treatment was 43.8 months (range 27-69). Patients alive at the time of evaluation were censored at the date of last follow-up (data log April 30, 2018). No patients were lost to follow-up. The $1-, 2-$, and 3-year OS rate was $75,42,33 \%$, respectively. Median OS was 23.9 months (range 1-49). The 1-, 2-, and 3 -year PFS rate was 42,8 , and $8 \%$, respectively. Median PFS was 10.1 months (range 2-40) (Fig. 3). Of the 8 patients with progressive disease, 4 had intra- and extrahepatic progression, 2 had extrahepatic progression only, and 2 had intrahepatic progression only.

\section{Post-Study Treatment}

After completion of the 3 months of study treatment, 8 patients (66.7\%) continued with gemcitabine/cisplatin for a median of 7.5 cycles in total (range 6-11), every 3 weeks, and HAI-FUDR for a median time of 5.5 months (range 4-26). Treatment was stopped due to disease progression $(n=3)$, pump complications that required a pump explantation (volvulus: $n=1$; pump dysfunction: $n=1)$, and other reasons $(n=3)$.

One patient had a tumor resection following 6 cycles of gemcitabine/cisplatin and HAI-FUDR, but achieving only R1 resection status with eventual tumor progression 1 year postoperatively. Another patient - after further second-line chemotherapy with irinotecan and HAIFUDR/mitomycin $\mathrm{C}$ and later radioembolization treatment - received a living donor liver transplant following hepatectomy and has remained in complete remission for 24 months until data cut-off. The patient who had the volvulus has remained progression free without treatment for 42 months until data cut-off.

\section{Discussion}

This study demonstrated the feasibility of the combination treatment of systemic gemcitabine/cisplatin with HAI-FUDR in patients with unresectable CCA with an acceptable toxicity profile. The treatment-related toxicity was comparable to that reported in previous studies with HAI treatment and systemic treatment for CCA. The most frequently seen higher-grade AEs were an increase in liver function test results, the occurrence of predominantly hepatobiliary infections, cytopenias due to the systemic treatment, and fatigue. The toxicities due to the study medication were well manageable with appropriate supportive measures as well as with a dose delay or reduction of the study medication.

Systemic and Intra-Arterial

Chemotherapy for Cholangiocarcinoma
The MTD for gemcitabine was defined as $800 \mathrm{mg} / \mathrm{m}^{2}$, which is $20 \%$ lower than usually administered in combination with cisplatin. We considered this reduction of one systemically administered drug acceptable, as our patients had no extrahepatic disease and the addition of HAI-FUDR to the doublet intensified the exposure of the liver tumors to cytotoxic agents compared to the fully dosed systemic doublet alone. Accordingly, we observed some considerable responses and long-term tumor control but also extrahepatic relapses irrespective of the administered gemcitabine dose, highlighting the importance of FUDR in this treatment strategy.

The partial response rate was $27 \%$, and the disease control rate $100 \%$. No patients experienced tumor progression within the 3 months. This finding compares favorably with the reported tumor control rates of about $80 \%$ with cisplatin and gemcitabine alone [5].

We also observed that only patients with intrahepatic CCA, but not those with hilar CCA, achieved significant responses to the study treatment. One obvious reason is the rather longitudinal growth of hilar CCA along the bile ducts and the difficulty to assess response based on morphological RECIST criteria compared to intrahepatic CCA. However, this finding could in part also be explained by the fact that the tumor biology of hilar bile duct carcinomas is different from that of carcinomas arising from the intrahepatic bile ducts, and may respond less to HAI and systemic treatment. Also, HAI chemotherapy is given via the hepatic artery and may thus cause toxic biliary sclerosis, as has been described in earlier studies [20, $21]$. We hypothesize that this toxic effect, which can be reduced by the additional administration of steroids, may render the affected bile ducts more prone to infectious complications than they already are due to the stenosing growth of the tumor, resulting in delay, reduction, or omission of effective treatment [20-22].

Although none of the patients achieved resectability after 3 months, the 3 -year OS rate was quite promising with 33\%. The median OS and PFS was 23.9 and 10.1 months, respectively. In 2 patients, long-term remission could be achieved, with disease stabilization in one patient as of data cut-off, while the other patient underwent successful liver transplantation after disease control for over 2 years. This outcome is quite pleasing, since all patients included had locally advanced and not primarily resectable disease, and one-third of the patients had locoregional nodal involvement. This finding is in line with large multi-institutional series showing nodal involvement in up to $37 \%$ of cases $[23,24]$. According to most experts, patients with locoregional nodal involvement 
may still be candidates for surgery, but should be approached with caution, considering chemotherapy first [25]. The survival we observed was in line with retrospective data on intra-arterial HAI-FUDR in CCA patients reported so far $[6,8]$. It has to be stated, however, that the prognosis may generally be more favorable in our study patients than in the patients included in the ABC-02 study, as our patients had no distant metastatic disease at diagnosis [26].

To date, no study has been published to our knowledge that has investigated the combination treatment of systemic chemotherapy with gemcitabine and cisplatin, which may be considered the current standard of practice, and intrahepatic chemotherapy with FUDR in patients with nonresectable CCA in a prospective fashion. Our results strengthen the notion that combining the current standard treatment with an efficacious third drug the latter given intra-arterially to avoid additional systemic toxicities - is feasible and may achieve promising results in selected patients, although downsizing to achieve resectability seems still challenging in this difficult-to-treat disease. We used a surgically implantable pump system to administer the intrahepatic agent, but also more conventional port systems, implanted by interventional radiologists and thus more broadly available and more convenient for the patients, could be used for this purpose [27].

\section{Conclusion}

Intravenous gemcitabine/cisplatin plus HAI-FUDR is feasible and appears effective for disease control in unresectable intrahepatic CCA. Our study may serve as the clinical basis for larger prospective studies evaluating this triplet combination.

\section{Statement of Ethics}

The trial was planned and conducted in accordance with the Declaration of Helsinki, the Guidelines for Good Clinical Practice issued by $\mathrm{ICH}$, and requirements of the respective national regulatory authorities. The study protocol and amendments were approved by the respective Regulatory Authorities and Ethics Committee of the Canton of Zurich (reference No. KEK-ZH 20110319). Written informed consent was obtained from all patients prior to enrollment.

\section{Conflict of Interest Statement}

The authors declare no conflicts of interest.

\section{Funding Sources}

This study was partly supported by a Grant of the University Hospital Zurich.

\section{Author Contributions}

H. Pietge analyzed and interpreted the patient data and was a major contributor in writing the manuscript. P.S. was the principal investigator and was a major contributor in writing the manuscript. P.S.-V. analyzed the surgical patient data and was a major contributor in writing the manuscript. A.S., T.W., S.B., A.K., H. Petrowsky, B.P., and P.-A.C. were main subinvestigators and enrolled the majority of the patients. H.B. was the clinical trial nurse responsible for patient data collection. D.A. did the statistical analysis. T.D.L.N.-K. did the radiological response assessment. All authors read and approved the final manuscript.

\section{Acknowledgements}

The authors would like to thank all patients who participated in the study.

\section{References}

1 Saha SK, Zhu AX, Fuchs CS, Brooks GA. Forty-year trends in cholangiocarcinoma incidence in the u.s.: intrahepatic disease on the rise. Oncologist. 2016;21(5):594-9.

2 Patel T. Increasing incidence and mortality of primary intrahepatic cholangiocarcinoma in the United States. Hepatology. 2001;33(6): 1353-7.

3 Khan SA, Davidson BR, Goldin R, Pereira SP, Rosenberg WM, Taylor-Robinson SD, et al. Guidelines for the diagnosis and treatment of cholangiocarcinoma: consensus document. Gut. 2002;51(Suppl 6):VI1-9.
4 Rosen CB, Heimbach JK, Gores GJ. Liver transplantation for cholangiocarcinoma. Transpl Int. 2010;23(7):692-7.

5 Valle J, Wasan H, Palmer DH, Cunningham D, Anthoney A, Maraveyas A, et al. Cisplatin plus gemcitabine versus gemcitabine for biliary tract cancer. N Engl J Med. 2010;362(14):1273-81.

6 Konstantinidis IT, Groot Koerkamp B, Do RK, Gönen M, Fong Y, Allen PJ, et al. Unresectable intrahepatic cholangiocarcinoma: systemic plus hepatic arterial infusion chemotherapy is associated with longer survival in comparison with systemic chemotherapy alone. Cancer. 2016;122(5):758-65.
7 Jarnagin WR, Schwartz LH, Gultekin DH Gönen M, Haviland D, Shia J, et al. Regional chemotherapy for unresectable primary liver cancer: results of a phase II clinical trial and assessment of DCE-MRI as a biomarker of survival. Ann Oncol. 2009;20(9):1589-95.

8 Cercek A, Boerner T, Tan BR, Chou JF, Gönen M, Boucher TM, et al. Assessment of hepatic arterial infusion of floxuridine in combination with systemic gemcitabine and oxaliplatin in patients with unresectable intrahepatic cholangiocarcinoma: a phase 2 clinical trial. JAMA Oncol. 2019; 6(1):60-67. 
9 Kuramoto K, Beppu T, Nitta H, Imai K, Masuda T, Miyata T, et al. Hepatic resection followed by hepatic arterial infusion chemotherapy for hepatocellular carcinoma with intrahepatic dissemination. Anticancer Res. 2018; 38(1):525-31.

10 He MK, Zou RH, Li QJ, Zhou ZG, Shen JX, Zhang YF, et al. Phase II study of sorafenib combined with concurrent hepatic arterial infusion of oxaliplatin, 5-fluorouracil and leucovorin for unresectable hepatocellular carcinoma with major portal vein thrombosis. Cardiovasc Intervent Radiol. 2018;41(5):73443.

11 Zervoudakis A, Boucher T, Kemeny NE. Treatment options in colorectal liver metastases: hepatic arterial infusion. Visc Med. 2017; 33(1):47-53.

12 Pernot S, Velut G, Kourie RH, Amouyal G, Sapoval M, Pointet AL, et al. 5-FU or mitomycin $\mathrm{C}$ hepatic arterial infusion after failure of arterial oxaliplatin in patients with colorectal cancer unresectable liver metastases. Clin Res Hepatol Gastroenterol. 2018;42(3):255-60.

13 Moriguchi M, Aramaki T, Nishiofuku H, Sato R, Asakura K, Yamaguchi K, et al. Sorafenib versus hepatic arterial infusion chemotherapy as initial treatment for hepatocellular carcinoma with advanced portal vein tumor thrombosis. Liver Cancer. 2017;6(4):275-86.
14 Kemeny NE, Chou JF, Boucher TM, Capanu M, DeMatteo RP, Jarnagin WR, et al. Updated long-term survival for patients with metastatic colorectal cancer treated with liver resection followed by hepatic arterial infusion and systemic chemotherapy. J Surg Oncol. 2016; 113(5):477-84.

15 Ensminger WD, Gyves JW. Clinical pharmacology of hepatic arterial chemotherapy. Semin Oncol. 1983;10(2):176-82.

16 Kemeny NE. Hepatic arterial therapy for the treatment of metastatic colorectal cancer. Semin Oncol. 2000;27(5 Suppl 10):126-31.

17 Ren Q, Kao V, Grem JL. Cytotoxicity and DNA fragmentation associated with sequential gemcitabine and 5-fluoro- 2 '-deoxyuridine in HT-29 colon cancer cells. Clin Cancer Res. 1998;4(11):2811-8

18 Dindo D, Demartines N, Clavien PA. Classification of surgical complications: a new proposal with evaluation in a cohort of $6336 \mathrm{pa}$ tients and results of a survey. Ann Surg. 2004; 240(2):205-13.

19 Slankamenac K, Nederlof N, Pessaux P, de Jonge J, Wijnhoven BP, Breitenstein S, et al. The comprehensive complication index: a novel and more sensitive endpoint for assessing outcome and reducing sample size in randomized controlled trials. Ann Surg. 2014; 260(5):757-62; discussion 762-3.

20 Kemeny N, Conti JA, Cohen A, Campana P, Huang Y, Shi WJ, et al. Phase II study of hepatic arterial floxuridine, leucovorin, and dexamethasone for unresectable liver metastases from colorectal carcinoma. J Clin Oncol. 1994;12(11):2288-95.
21 Kemeny N, Seiter K, Niedzwiecki D, Chapman D, Sigurdson E, Cohen A, et al. A randomized trial of intrahepatic infusion of fluorodeoxyuridine with dexamethasone versus fluorodeoxyuridine alone in the treatment of metastatic colorectal cancer. Cancer. 1992; 69(2):327-34.

22 Jarnagin W, Winston C. Hilar cholangiocarcinoma: diagnosis and staging. HPB (Oxford). 2005;7(4):244-51.

23 Ribero D, Pinna AD, Guglielmi A, Ponti A, Nuzzo G, Giulini SM, et al. Surgical approach for long-term survival of patients with intrahepatic cholangiocarcinoma: a multi-institutional analysis of 434 patients. Arch Surg. 2012;147(12):1107-13

24 de Jong MC, Nathan H, Sotiropoulos GC, Paul A, Alexandrescu S, Marques H, et al. Intrahepatic cholangiocarcinoma: an international multi-institutional analysis of prognostic factors and lymph node assessment. J Clin Oncol. 2011;29(23):3140-5.

25 Ebata T, Ercolani G, Alvaro D, Ribero D, Di Tommaso L, Valle JW. Current status on cholangiocarcinoma and gallbladder cancer. Liver Cancer. 2016;6(1):59-65.

26 Kim BJ, Hyung J, Yoo C, Kim KP, Park SJ, Lee SS, et al. Prognostic factors in patients with advanced biliary tract cancer treated with first-line gemcitabine plus cisplatin: retrospective analysis of 740 patients. Cancer Chemother Pharmacol. 2017;80(1):209-15.

27 Callahan MK, Kemeny NE. Implanted hepatic arterial infusion pumps. Cancer J. 2010; 16(2):142-9. 